\title{
TENSOR PRODUCTS OF BANACH ALGEBRAS. II
}

\author{
BERNARD R. GELBAUM ${ }^{1}$
}

0 . In [1], [2] there are descriptions of an alleged bijection $\mathfrak{N}_{3}$ $\leftrightarrow \mathfrak{M}_{1} \times \mathfrak{T}_{2}$, where $\mathfrak{M}_{i}$ is the set of regular maximal ideals of the Banach algebra $A_{i}, i=1,2,3$, and where $A_{3}=A_{1} \otimes_{\gamma} A_{2}$ is the greatest cross-norm tensor product of $A_{1}$ and $A_{2}$. The given constructions for the bijection are valid if both $A_{1}$ and $A_{2}$ are commutative. Otherwise, the constructions are in error (e.g., [1, p. 301, line 12]; [2, p. 753, line 4 and p. 755, Case 2]). The author knows of no counterexamples to the conclusions in [1], [2], [3].

The purpose of this note is to offer (correct) proofs of modified results in more general contexts and to apologize for the regrettable oversights. New research of K. Laursen, whose work (in prepublished form) the author had the privilege to examine, is based on the existence of the bijection mentioned above. Exposure to the new research led to the author's discovery of his earlier errors.

1. Let $A_{1}, A_{2}$ be (not necessarily commutative) Banach algebras with identities $e_{1}$ and $e_{2}$. Let $A_{3}=A_{1} \otimes_{\gamma} A_{2}$ (with identity $e_{1} \otimes e_{2}$ ). For $i=1,2,3$ let $\mathscr{L}_{i}$ denote the set of proper closed left ideals in $A_{i}$. We define two maps:

$$
T: \mathfrak{L}_{1} \times \mathfrak{L}_{2} \rightarrow \mathfrak{L}_{3}, \quad S: \mathfrak{L}_{3} \rightarrow \mathfrak{L}_{1} \times \mathfrak{L}_{2}
$$

as follows:

$$
\begin{aligned}
& \text { ad } T: \text { If } L_{i} \in \mathscr{L}_{i}, \quad i=1,2, \quad \text { define the linear map } \\
& R: A_{3} \ni a_{3}=\sum_{n} a_{1 n} \otimes a_{2 n} \rightarrow \sum_{n}\left(a_{1 n} / L_{1}\right) \otimes\left(a_{2 n} / L_{2}\right) \\
& \in\left(A_{1} / L_{1}\right) \otimes_{\gamma}\left(A_{2} / L_{2}\right) .
\end{aligned}
$$

Then $\operatorname{ker}(R) \equiv T\left(L_{1}, L_{2}\right) \in \mathscr{L}_{3}$.

$$
\begin{aligned}
& \text { ad } S: \text { If } L_{3} \in \mathscr{L}_{3} \text { define the linear maps } \\
& G_{1}: A_{1} \ni a_{1} \rightarrow\left(a_{1} \otimes e_{2}\right) / L_{3} \in A_{3} / L_{3}, \\
& G_{2}: A_{2} \ni a_{2} \rightarrow\left(e_{1} \otimes a_{2}\right) / L_{3} \in A_{3} / L_{3} .
\end{aligned}
$$

Then $\operatorname{ker}\left(G_{i}\right) \equiv L_{i} \in \mathscr{L}_{i}, i=1,2$. We set $S\left(L_{3}\right)=\left(L_{1}, L_{2}\right)$. (Alternatively: $S\left(L_{3}\right)=\left(L_{3} \cap\left(A_{1} \otimes e_{2}\right), L_{3} \cap\left(e_{1} \otimes A_{2}\right)\right)$.)

Received by the editors January 28, 1969 and, in revised form, November 10, 1969.

1 This research was supported in part by National Science Foundation Grant GP5436 for which the author is grateful. 
Lemma 1. Let $L_{3} \subset L_{3}^{\prime}, S\left(L_{3}\right)=\left(L_{1}, L_{2}\right), S\left(L_{3}^{\prime}\right)=\left(L_{1}^{\prime}, L_{2}^{\prime}\right)$. Then $L_{i} \subset L_{i}^{\prime}, i=1,2$.

Proof. Reference to the alternative definition of $S$ leads directly to the result.

Lemma 2. If $L_{1} \subset L_{1}^{\prime}, L_{2} \subset L_{2}^{\prime}, T\left(L_{1}, L_{2}\right)=L_{3}, T\left(L_{1}^{\prime}, L_{2}^{\prime}\right)=L_{3}^{\prime}$, then $L_{3} \subset L_{3}^{\prime}$. If $L_{1} \subsetneq L_{1}^{\prime}$ or $L_{2} \neq L_{2}^{\prime}$ then $L_{3} \neq L_{3}^{\prime}$.

Proof. Let $E_{i}: A_{i} \rightarrow A_{i} / L_{i}, E_{i}^{\prime}: A_{i} \rightarrow A_{i} / L_{i}^{\prime}$ be the canonical maps, $i=1,2$. We may define a third map $F_{i}: A_{i} / L_{i} \rightarrow A_{i} / L_{i}^{\prime}, i=1,2$, as follows. If $y \in A_{i} / L_{i}$, let $E_{i}(x)=y$ and let $F_{i}(y)=E_{i}^{\prime}(x)$. If $E_{i}(\tilde{x})=y$ then $\tilde{x}-x \in L_{i} \subset L_{i}^{\prime}$ whence $E_{i}^{\prime}(\tilde{x})=E_{i}(x)$ and thus $F_{i}(y)$ is well defined. Furthermore, if $z=E_{i}^{\prime}(x)$ then $F_{i} E_{i}(x)=z$ and thus the following diagram

$$
\begin{array}{r}
\stackrel{E_{i}}{A_{i}} A_{i} / L_{i} \\
E_{i}^{\prime} \searrow \downarrow F_{i} \\
A_{i} / L_{i}^{\prime}
\end{array}
$$

$i=1,2$, is commutative. If $R^{\prime}: A_{3} \rightarrow\left(A_{1} / L_{1}^{\prime}\right) \otimes_{\gamma}\left(A_{2} / L_{2}^{\prime}\right)$, then

$$
\begin{aligned}
R^{\prime}\left(\sum_{n} a_{1 n} \otimes a_{2 n}\right) & =\sum_{n}\left(a_{1 n} / L_{1}^{\prime}\right) \otimes\left(a_{2 n} / L_{2}^{\prime}\right) \\
& =\sum_{n} E_{1}^{\prime}\left(a_{1 n}\right) \otimes E_{2}^{\prime}\left(a_{2 n}\right) \\
& =\left(F_{1} \otimes F_{2}\right) \sum_{n} E_{1}\left(a_{1 n}\right) \otimes E_{2}\left(a_{2 n}\right) \\
& =F_{1} \otimes F_{2} \circ R\left(\sum_{n} a_{1 n} \otimes a_{2 n}\right) .
\end{aligned}
$$

Thus $\operatorname{ker}(R)=T\left(L_{1}, L_{2}\right) \subset \operatorname{ker}\left(R^{\prime}\right)=T\left(L_{1}^{\prime}, L_{2}^{\prime}\right)$. If either $L_{1 \neq} \subsetneq L_{1}^{\prime}$ or $L_{2} \subsetneq L_{2}^{\prime}$ then correspondingly one of $\operatorname{ker}\left(F_{1}\right), \operatorname{ker}\left(F_{2}\right)$ is nontrivial. Thus, e.g., if $0 \neq E_{1}\left(a_{1}\right) \in \operatorname{ker}\left(F_{1}\right), \quad E_{2}\left(a_{2}\right) \neq 0$, we find $a_{1} \otimes a_{2} \in$ $\operatorname{ker}\left(R^{\prime}\right) \backslash \operatorname{ker}(R)$.

We shall consider together with $\mathfrak{L}_{i}$ the sets $\mathfrak{M}_{l i}, \mathscr{I}_{i}, \mathfrak{T}_{i}$ consisting respectively of maximal left ideals, closed ideals and maximal ideals in $A_{i}, i=1,2,3$.

Lemma 3. (a) $T\left(\mathscr{g}_{1} \times \mathscr{g}_{2}\right) \subset \mathfrak{g}_{3}$;

(b) $S\left(g_{3}\right) \subset g_{1} \times g_{2}$;

(c) if $\left(J_{1}, J_{2}\right)$ belongs to any of $\mathfrak{L}_{1} \times \mathfrak{L}_{2}, \mathfrak{T}_{l 1} \times \mathfrak{T}_{l 2}, \mathfrak{g}_{1} \times \mathfrak{g}_{2}$ or $\mathfrak{M}_{1} \times \mathbb{T}_{2}$ and if $S T\left(J_{1}, J_{2}\right)=\left(J_{1}^{\prime}, J_{2}^{\prime}\right)$, then $J_{i}^{\prime} \supset J_{i}, i=1,2$. 
Proof. The assertions (a) and (b) follow immediately from the relevant definitions. For (c) we note that if $a_{1} \in J_{1}$, then $a_{1} / J_{1}=0$ whence $a_{1} \otimes e_{2} \in T\left(J_{1}, J_{2}\right) \equiv J_{3}$ wherice $a_{1} \in J_{1}^{\prime}$. Thus $J_{1} \subset J_{1}^{\prime}$ and by a similar argument $J_{2} \subseteq J_{2}^{\prime}$.

Corollary. On $\mathfrak{M}_{l 1} \times \mathfrak{M}_{l 2}$ and on $\mathfrak{M}_{1} \times \mathbb{T}_{2}$ the map $S T=$ identity.

For each $I_{i} \in g_{i}$ let $I_{i}$ be some arbitrary but fixed element of $\mathfrak{M}_{i}$ and assume $\tilde{I}_{\boldsymbol{i}} \supset I_{\boldsymbol{i}}$. Define mappings $\widetilde{S}$ and $\widetilde{T}$ as follows:

$$
\tilde{S}: \mathscr{I}_{8} \ni I_{3} \rightarrow \widetilde{S}\left(I_{3}\right) \equiv\left(\tilde{I}_{1}, \tilde{I}_{2}\right)
$$

where $S\left(I_{3}\right)=\left(I_{1}, I_{2}\right)$.

$$
\tilde{T}: \mathscr{I}_{1} \times \mathscr{I}_{2} \ni\left(I_{1}, I_{2}\right) \rightarrow \tilde{T}\left(I_{1}, I_{2}\right)=I_{3}
$$

where $T\left(I_{1}, I_{2}\right)=I_{3}$. Despite the arbitrariness in the definitions of $\tilde{S}$ and $\tilde{T}$ we have

TheOREM 1. On $\mathfrak{T}_{1} \times \mathfrak{T}_{2}, \tilde{S} \tilde{T}=$ identity. If hk (hull-kernel) topologies are used throughout then $\tilde{S}$ is a continuous bijection of $\widetilde{T}\left(\mathfrak{N}_{1} \times \mathfrak{T C}_{2}\right)$ on $\mathfrak{M}_{1} \times \mathbb{N}_{2}$. (Note that $\tilde{T}\left(\mathfrak{N}_{1} \times \mathfrak{M}_{2}\right) \subset \mathbb{N}_{3}$.)

Proof. Since $S T=$ identity on $\mathfrak{M}_{1} \times \mathbb{N}_{2}$ we see $S$ is a bijection of $T\left(\mathfrak{T}_{1} \times \mathfrak{T}_{2}\right)$ onto $\mathfrak{T}_{1} \times \mathfrak{T C}_{2}$. The parallel argument here reads: $T\left(M_{1}, M_{2}\right)=I_{3} \subset \tilde{I}_{3}=\widetilde{T}\left(M_{1}, M_{2}\right)$. By Lemma $1, S$ is inclusion preserving. If $S\left(I_{3}\right)=\left(M_{1}, M_{2}\right)$ and $S\left(\tilde{I}_{3}\right)=\left(I_{1}, I_{2}\right)$ then $I_{3} \subset \tilde{I}_{3}$ implies $M_{i} \subset I_{i}$ and so $M_{i}=I_{i}$ since $M_{i}$ is maximal, $i=1,2$. Thus $\tilde{S} \widetilde{T}\left(M_{1}, M_{2}\right)$ $=\widetilde{S}\left(\tilde{I}_{3}\right)=\left(\tilde{I}_{1}, \tilde{I}_{2}\right)=\left(M_{1}, M_{2}\right)$. Hence $\widetilde{S} \widetilde{T}=$ identity on $\mathfrak{M}_{1} \times \mathfrak{T}_{2}$ and hence $\widetilde{T}$ is injective; consequently $\widetilde{S}$, on $\widetilde{T}\left(\mathscr{T}_{1} \times \mathscr{T}_{2}\right)$, is bijective.

If $F_{1}$ is closed in $\mathfrak{T}_{1}$ then we show $\widetilde{T}\left(F_{1} \times \Re_{2}\right)$ is closed in $\widetilde{T}\left(\mathfrak{T}_{1} \times \mathfrak{N}_{2}\right)$. A similar proof shows that if $F_{2}$ is closed in $\mathfrak{N}_{2}$ then $\tilde{T}\left(\mathscr{T}_{1} \times F_{2}\right)$ is closed in $\tilde{T}\left(\mathscr{T C}_{1} \times \mathbb{N}_{2}\right)$. Since $\tilde{T}$ is injective and since any closed set in $\mathfrak{M T}_{1} \times \mathbb{N}_{2}$ is of the form

$$
\bigcap_{\gamma}\left[\left(F_{1}^{\gamma} \times \mathscr{M}_{2} \cup \mathfrak{M}_{1} \times F_{2}^{\gamma}\right)\right]
$$

where $F_{i}^{\gamma}$ is closed in $\mathfrak{N}_{i}, i=1,2$, we see that

$$
\widetilde{T}\left(\bigcap_{\gamma}\left[\left(F_{1}^{\gamma} \times \mathscr{M}_{2}\right) \cup\left(\mathscr{T}_{1} \times F_{2}^{\gamma}\right)\right]\right)=\bigcap_{\gamma}\left[\widetilde{T}\left(F_{1}^{\gamma} \times \mathscr{T}_{2}\right) \cup \tilde{T}\left(\mathscr{T}_{1} \times F_{2}^{\gamma}\right)\right]
$$

is closed. Furthermore $\tilde{T}: \mathfrak{T}_{2} \times \mathfrak{T}_{1} \leftrightarrow \widetilde{T}\left(\mathfrak{T}_{1} \times \mathfrak{T}_{2}\right)$ is closed, $\tilde{S}: \tilde{T}\left(\mathscr{T}_{1} \times \mathbb{M}_{2}\right) \leftrightarrow \mathbb{M}_{1} \times \mathbb{T}_{2}$ is $\tilde{T}^{-1}$ and thus $\tilde{S}$ is continuous. (This proof is essentially that given in [2]. There a counterexample shows $\widetilde{T}$ need not be continuous.)

Thus let $M_{3}^{0}=\tilde{T}\left(M_{1}^{0}, M_{2}^{0}\right) \supset k\left[\tilde{T}\left(F_{1} \times \mathbb{T}_{2}\right)\right]$. A contradiction will be 
obtained by constructing a single element $a_{1}^{0} \otimes a_{2}^{0} \in \tilde{T}\left(M_{1}, M_{2}\right) \backslash M_{3}^{0}$ for all $\left(M_{1}, M_{2}\right) \in F_{1} \times \mathscr{T}_{2}$. Thus $a_{1}^{0} \otimes a_{2}^{0} \in k\left[\widetilde{T}\left(F_{1} \times \mathscr{T}_{2}\right)\right]$.

If $M_{3}^{0} \notin \tilde{T}\left(F_{1} \times T_{2}\right)$ then $M_{1}^{0} \notin F_{1}$ and thus $M_{1}^{0} D k\left(F_{1}\right)$. Let $a_{1}^{0}$ $\in k\left(F_{1}\right) \backslash M_{1}^{0}$. Then $a_{1}^{0} / M_{1}^{0} \neq 0$. On the other hand, for $a_{2} \in A_{2}$ consider $\left(a_{1}^{0} \otimes a_{2}\right) / M_{3}$ where $M_{3}=\widetilde{T}\left(M_{1}, M_{2}\right),\left(M_{1}, M_{2}\right) \in F_{1} \times \mathfrak{T}_{2}$. Then since $a_{1}^{0} \in k\left(F_{1}\right) \subset M_{1}$ we see $\left(a_{1}^{0} / M_{1}\right) \otimes\left(a_{2} / M_{2}\right)=0$, i.e., $a_{1}^{0} \otimes a_{2} \in M_{3}$. Since $\left(M_{1}, M_{2}\right)$ are arbitrary in $F_{1} \times \Re_{2}$ we conclude $a_{1}^{0} \otimes a_{2} \in k\left[\tilde{T}\left(F_{1} \times \Re_{2}\right)\right]$ $\subset M_{3}^{0}$. Hence $\left(a_{1}^{0} \otimes a_{2}\right) / M_{3}^{0}=0$. Now choose $a_{2}^{0} \notin M_{2}^{0}$ (whence $\left.a_{2}^{0} / M_{2}^{0} \neq 0\right)$. Then $\left(a_{1}^{0} / M_{1}^{0}\right) \otimes\left(a_{2}^{0} / M_{2}^{0}\right) \neq 0$ and so $a_{1}^{0} \otimes a_{2}^{0} \notin M_{3}^{0}=\widetilde{T}\left(M_{1}^{0}, M_{2}^{0}\right)$, a contradiction.

The argument in $[3$, p. 538, line 3 through p. 539, line 7] depends on the bijection $\mathfrak{M}_{1} \times \mathfrak{M}_{2} \leftrightarrow \mathfrak{M}_{3}$ alleged and discussed in [2]. Although the author knows of no instance where the bijection is absent, he is unaware of correct proofs. In what follows $\lambda$ is the "least cross-norm whose associate is a cross-norm" [4, pp. 30-36] and $\tau$ is the extension of the identity map from the algebraic tensor product to the completions: $A_{1} \otimes_{\gamma} A_{2} \equiv A_{3} \rightarrow A_{1} \otimes_{\lambda} A_{2}$.

Theorem 2. Let $A_{1}$ and $A_{2}$ be strongly semisimple $\left(\cap_{\mathfrak{N} \tau_{i}} M_{i}=\{0\}\right.$, $i=1,2)$ and assume that the map $\tau: A_{1} \otimes_{\gamma} A_{2} \equiv A_{3} \rightarrow A_{1} \otimes_{\lambda} A_{2}$ is 1-1. Furthermore assume that spectral synthesis holds for each element of $T\left(\mathscr{T}_{1} \times \mathfrak{T l}_{2}\right)$, i.e., that $k h\left(T\left(M_{1}, M_{2}\right)\right)=T\left(M_{1}, M_{2}\right)$ for all $\left(M_{1}, M_{2}\right)$ in $\mathfrak{M}_{1} \times \mathscr{T}_{2}$. Then $\cap\left\{M_{3}: M_{3} \in \bigcup_{\mathfrak{N}_{1} \times \mathbb{N}_{2}} h\left(T\left(M_{1}, M_{2}\right)\right)\right\} \equiv P=\{0\}$ and in particular $A_{3}$ is strongly semisimple since $\bigcap_{\mathfrak{N}_{3}} M_{3} \subset P=\{0\}$.

Proof. Let $a_{3} \in \cap\left\{M_{3}: M_{3} \in \mathrm{U}_{\mathbb{N}_{1} \times \Re_{2}} h\left(T\left(M_{1}, M_{2}\right)\right)\right\}$. Then in particular $a_{3} \in k h\left(T\left(M_{1}, M_{2}\right)\right)$ for all $\left(M_{1}, M_{2}\right) \in \mathfrak{M}_{1} \times \mathfrak{M}_{2}$. By hypothesis, $a_{3} \in T\left(M_{1}, M_{2}\right)$ for all $\left(M_{1}, M_{2}\right) \in \mathfrak{M}_{1} \times \mathfrak{M}_{2}$. Hence, if $a_{3}=\sum_{n} a_{1 n} \otimes a_{2 n}$ then

$$
\sum_{n}\left(a_{1 n} / M_{1}\right) \otimes\left(a_{2 n} / M_{2}\right)=0
$$

for all $\left(M_{1}, M_{2}\right) \in 9 \pi_{1} \times \Re_{2}$. If $a_{3} \neq 0$ then $\tau\left(a_{3}\right) \neq 0$ and thus $0<\lambda\left(a_{3}\right)$ $=\sup \left\{\left|\sum_{n} f_{1}\left(a_{1 n}\right) f_{2}\left(a_{2 n}\right)\right|:\left(f_{1}, f_{2}\right) \in A_{1}^{*} \times A_{2}^{*},\left|f_{1}\right|=\left|f_{2}\right|=1\right\}$. This for some $\left(f_{1}, f_{2}\right) \in A_{1}^{*} \times A_{2}^{*}$ there obtains

$$
\sum_{n} f_{1}\left(a_{1 n}\right) f_{2}\left(a_{2 n}\right) \neq 0 .
$$

We find in turn:

$$
\sum_{n} f_{1}\left(a_{1 n}\right) a_{2 n} \neq 0
$$

for some $M_{2}^{0}$ 


$$
\sum_{n} f_{1}\left(a_{1 n}\right) a_{2 n} / M_{2}^{0} \neq 0
$$

since $A_{2}$ is semisimple;

$$
\sum_{n} f_{1}\left(a_{1 n}\right) \psi_{2}\left(a_{2 n} / M_{2}^{0}\right) \neq 0
$$

for some $\psi_{2} \in\left(A_{2} / M_{2}^{0}\right)^{*}$;

$$
\sum_{n} \psi_{2}\left(a_{2 n} / M_{2}^{0}\right) a_{1 n} \neq 0
$$

for some $M_{1}^{0}$

$$
\sum_{n} \psi_{2}\left(a_{2 n} / M_{2}^{0}\right)\left(a_{1 n} / M_{1}^{0}\right) \neq 0
$$

since $A_{1}$ is semisimple. Thus for some $\psi_{1} \in\left(A_{1} / M_{1}^{0}\right)^{*}$

$$
\sum_{n} \psi_{1}\left(a_{1 n} / M_{1}^{0}\right) \psi_{2}\left(a_{2 n} / M_{2}^{0}\right) \neq 0 \text {. }
$$

Hence $\quad 0<\lambda\left(\sum_{n}\left(a_{1 n} / M_{1}^{0}\right) \otimes\left(a_{2 n} / M_{2}^{0}\right)\right) \leqq \gamma\left(\sum_{n}\left(a_{1 n} / M_{1}^{0}\right) \otimes\left(a_{2 n} / M_{2}^{0}\right)\right)$, a contradiction of $\sum_{n}\left(a_{1 n} / M_{1}\right) \otimes\left(a_{2 n} / M_{2}\right)=0$ for all $\left(M_{1}, M_{2}\right)$ $\in \mathscr{T}_{1} \times \mathbb{N}_{2}$.

The appearance of spectral synthesis in the above is of interest. Indeed, the basic and, as far as the author knows, unresolved question about the semisimplicity of $A_{1} \otimes_{\gamma} A_{2}$ when $A_{1}$ and $A_{2}$ are semisimple (even commutative) is equivalent to spectral synthesis for the (reducing) ideal $I$ of $F_{\gamma}\left(A_{1}, A_{2}\right)$ [3, pp. 526, 540]. Of course, semisimplicity of any Banach algebra $A$ is the statement that spectral synthesis holds for the zero ideal $\{0\}$.

\section{BIBLIOGRAPHY}

1. B. R. Gelbaum, Tensor products of Banach algebras, Canad. J. Math. 11 (1959), 297-310. MR 21 \#2922.

2. - Note on the tensor product of Banach algebras, Proc. Amer. Math. Soc. 12 (1961), 750-757. MR 24 \#A1039.

3. - Tensor products and related questions, Trans. Amer. Math. Soc. 103 (1962), 525-548. MR 25 \#2406.

4. R. Schatten, $A$ theory of cross-spaces, Ann. of Math. Studies, no. 26, Princeton Univ. Press, Princeton, N. J., 1950. MR 12, 186.

University of California, Irvine, California 92664 